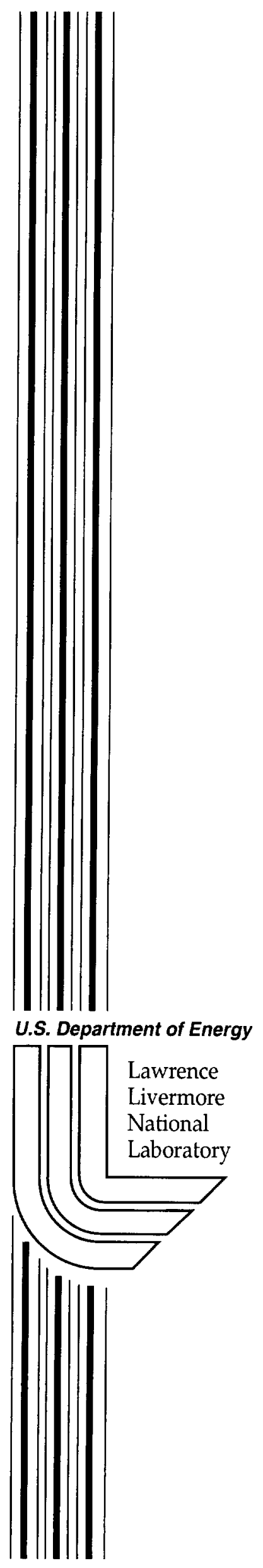

UCRL-ID-140596

\title{
Wireless Link Design Using A Patch Antenna
}

\author{
E. Hall
}

August 11, 2000 


\section{DISCLAIMER}

This document was prepared as an account of work sponsored by an agency of the United States Government. Neither the United States Government nor the University of California nor any of their employees, makes any warranty, express or implied, or assumes any legal liability or responsibility for the accuracy, completeness, or usefulness of any information, apparatus, product, or process disclosed, or represents that its use would not infringe privately owned rights. Reference herein to any specific commercial product, process, or service by trade name, trademark, manufacturer, or otherwise, does not necessarily constitute or imply its endorsement, recommendation, or favoring by the United States Government or the University of California. The views and opinions of authors expressed herein do not necessarily state or reflect those of the United States Government or the University of California, and shall not be used for advertising or product endorsement purposes.

This work was performed under the auspices of the U. S. Department of Energy by the University of California, Lawrence Livermore National Laboratory under Contract No. W-7405-Eng-48.

This report has been reproduced directly from the best available copy.

Available electronically at http://www.doc.gov/bridge

Available for a processing fee to U.S. Department of Energy

And its contractors in paper from

U.S. Department of Energy

Office of Scientific and Technical Information

P.O. Box 62

Oak Ridge, TN 37831-0062

Telephone: (865) 576-8401

Facsimile: (865) 576-5728

E-mail: reports@adonis.osti.gov

Available for the sale to the public from

U.S. Department of Commerce

National Technical Information Service

5285 Port Royal Road

Springfield, VA 22161

Telephone: (800) 553-6847

Facsimile: (703) 605-6900

E-mail: orders@ntis.fedworld.gov

Online ordering: http://www.ntis.gov/ordering.htm

\section{OR}

Lawrence Livermore National Laboratory

Technical Information Department's Digital Library

http://www.llnl.gov/tid/Library.html 


\title{
Wireless Link Design Using a Patch Antenna
}

\author{
Eric Hall
}

\begin{abstract}
A wireless link was designed using a patch antenna. In the process, several different models were tested. Testing proved a patch antenna was a viable solution for building a wireless link within the design specifications. Also, this experimentation provided a basis for future patch antenna design.
\end{abstract}

\section{Introduction}

As part of a digital telemetry system, a wireless link needed to be designed. This link was to be capable of transmitting through two inches of plastic (dielectric constant of 4.0) such as might be used in rotating machinery. Both transmitting and receiving antenna were to be as small as possible, and flush with their corresponding surfaces. Specifications required that the digital link be low power and capable of handling a maximum data rate of $10 \mathrm{Mbps}$. Also, the link needed to be able to withstand the effects of an electromagnetic pulse (EMP).

The requirement of withstanding an EMP was easily satisfied. Most of the energy from an EMP is distributed throughout the lower frequencies ranging between $3 \mathrm{~Hz}$ and $30 \mathrm{kHz}$. Data in this digital telemetry system was to be modulated onto a carrier at $2205.5 \mathrm{MHz}$. An antenna operating at this high frequency would not accept the lower frequency EMP. Thus the EMP would have a minimal effect upon the link.
To reduce power requirements of the system, transmitter and receiver devices were eliminated. Both devices used amplifiers to boost the power of the signal. An efficient antenna was expected to compensate for the absence of the power amplifiers, and still maintain a high data rate with low bit error.

The remaining design requirements were dependent upon the antenna element of the link. The antennas were to be small enough to be set within a milled out piece of metal about $5 \mathrm{~mm}$ thick. Also, the antenna needed to be flat and contained within the milled out area, and flush with the metal surface. In addition, the antenna needed to be easily fabricated. Finally, efficiency needed consideration. Efficiency had to be such, that despite the lack of power amplifiers to boost the signal before and after transmission, a low bit error rate could still be maintained. The patch antenna model was selected as best meeting the specifications of the link. This class of antenna was small enough to fit within a milled out space. Also, it was flat and would be flush with corresponding surfaces of the telemetry system. Assuming that the antenna would be satisfactorily efficient, all other specifications for design would be satisfied. To answer the efficiency concern, several models were made and tested to determine the feasibility of the patch antenna link. 
In order to test the wireless link, two large aluminum plates were fitted with coaxial cable input jacks (figure 1). Placed between the plates was a twoinch stack of paper serving as the dielectric medium through with the signal would be transmitted. The paper had a dielectric constant of 4 , equal to the dielectric constant of the plastic in project specifications. Next a network analyzer was used to measure the efficiency of the simple link.

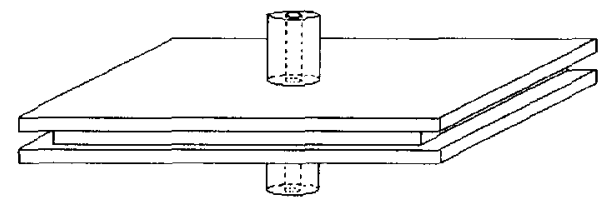

Figure 1: Test Plates Fitted with Coaxial Input Nodes

\section{The Patch Antenna}

Square patch antennas consist of a thin square of conductor material stacked closely above a larger reflector plate (figure 2). Between the patch element and the reflector plate is a dielectric element. The upper patch element measures roughly .5 wavelengths on each side. This active patch element functions similarly to two stacked dipoles, each .5 wavelengths in length, backed by a reflector plate. In actuality, the antenna is more similar to two slot antennas. The active patch element is connected to a transmission line at a feed point. This point can be anywhere from the center of the square surface, to the center of an edge. As the feed position moves outward, the antenna impedance increases.

As specified, this antenna would be small and flush. The active element was to be made using a thin conductive copper tape about 1 mil thick. The aluminum plate would serve as the reflector plate, thus not adding any size to the antenna. The largest element would be the dielectric element between the patch and reflector. This element, however, would be limited to a few mils in thickness. Thus, it would be possible for a section of the plate to be carved out, and the dielectric and patch to be set within. Also, all three layers would be flat and consequently flush with the existing equipment. Furthermore, the patch antenna would radiate in the correct direction, where a transmitted signal would be received by another patch antenna. Finally, these patches would be relatively easy to fabricate. Special equipment would not need to be employed in their production.

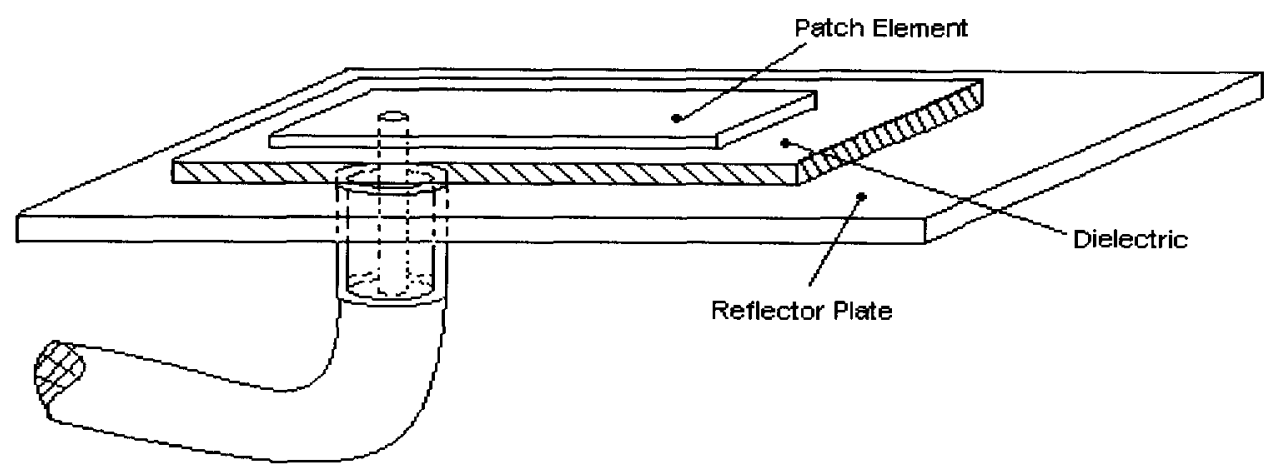


Efficiency of the antenna was the only parameter that required further exploration. It needed to be determined if the patch antenna would be efficient enough to insure a low bit error rate of transmission despite the absence of power amplifiers in the telemetry system. The patch antenna was tested using the aluminum plate apparatus described above.

\section{Measurements of Interest}

Before it was possible to optimize the antenna, optimal antenna operating conditions needed to be determined. This would occur when the antenna load impedance was equal to the transmission line impedance, the standing wave ratio (SWR) was equal to 1 , and the resonant frequency was equal to the carrier frequency.

Standing waves resulted in the transmission line when an impedance mismatch in the antenna caused energy to be reflected and lost. This reflected energy was measured as return loss, and resulted in decreased efficiency. The impedance of the coaxial transmission line was $50 \Omega$. When the impedance of the antenna was matched at $50 \Omega$, and there was no imaginary impedance, the SWR was equal to 1 , and performance was optimized. Spacing between the patch and reflector resulted in a capacitive effect. Likewise, an inductive effect occurred from current in the antenna connection. Ideally, capacitive and inductive effects would balance each other resulting in zero imaginary impedance.

The network analyzer measured return loss. Because capacitive effects varied with frequency, antenna impedance also varied. The analyzer frequency scan produced a spike when the line and load impedance were closely matched (figure 3). The height of the spike increased as matching was more exact. Optimized return loss would be $-\infty \mathrm{dB}$; however, a loss of less than $-10 \mathrm{~dB}$ would represent less than $10 \%$ loss in power due to load mismatch.

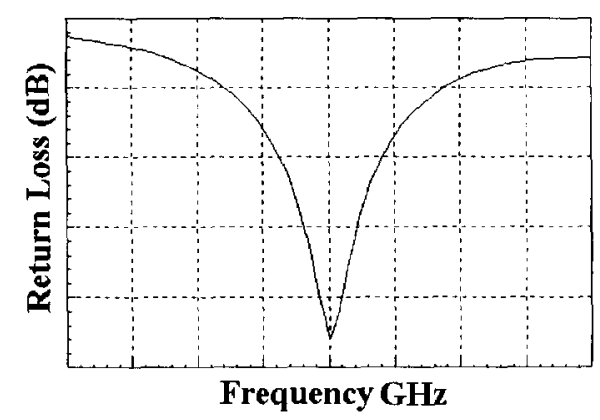

Figure 3: Return Loss Peaking at the Resonant Frequency

The frequency of optimization was the antenna's resonant frequency. The only frequency of concern was the one that was to be transmitted, $2250.5 \mathrm{MHz}$. Therefore, the antenna was to be optimized for transmitting the carrier frequency. The spike in the return loss measurements needed to be at $2250.5 \mathrm{MHz}$ for optimized results.

Most of the loss in the wireless link occurred in the waveform channel as the signal was being transmitted. An efficient antenna would minimize transmission loss. The network analyzer measured the transmission strength as well (figure 4). Since transmission strength depended upon load matching, strength of the transmission also was frequency dependant. An optimized antenna would have a transmission strength of $0 \mathrm{~dB}$ representing no loss. A lossless system, however, would 
represent a short between the two antennas. The antenna to be designed needed to have the strongest transmission at the carrier frequency. This was automatically accomplished by first establishing the minimal return loss point at the desired frequency.

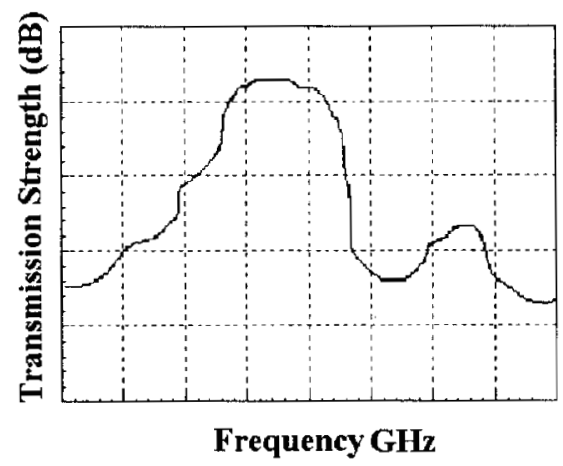

Figure 4: Transmission Strength Maximized at the Resonant Frequency

In order to eliminate any effects of the transmission lines or other elements unrelated to the antenna, the network analyzer was carefully calibrated before any measurements were taken so that only the link's effects would be measured.

\section{Starting Point}

In order to establish a basis for measuring improvement, the link's performance was first measured using the simplest antenna possible. This antenna was made by terminating the coaxial transmission line at the

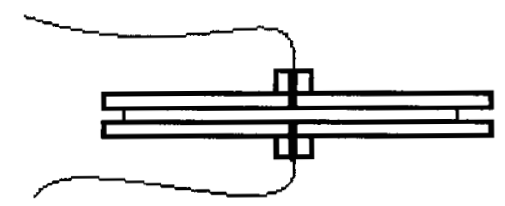

Figure 5: Coaxial Antenna Only connector port in the aluminum plate. The signal was transmitted from this straight terminated coaxial line without any patch element (figure 5). The coaxial antenna was measured at a frequency of $2250.5 \mathrm{MHz}$ yielding the results in table 1 .

\begin{tabular}{|c|c|}
\hline $\begin{array}{c}\text { Standing Wave } \\
\text { Ratio (SWR) }\end{array}$ & 4.1 \\
\hline Return Loss & $-4.0 \mathrm{~dB}$ \\
\hline $\begin{array}{c}\text { Antenna Load } \\
\text { Impedance }\end{array}$ & $126.4-\mathrm{j} 93.7 \Omega$ \\
\hline $\begin{array}{c}\text { Transmission } \\
\text { Strength }\end{array}$ & $-61.4 \mathrm{~dB}$ \\
\hline
\end{tabular}

Table 1: Coaxial Antenna Measurements@2250.5 MHz

These values revealed that this method of transmission was inefficient and unacceptable. For the transmission to be improved, the two antennas needed to be better matched. These measurements established a starting point against which improvements in transmission could be evaluated.

\section{Design Process Using G-10 Dielectric}

For the first attempt at using a patch antenna, a piece of $4 \mathrm{~mm}$ thick G-10 epoxy board was chosen for the dielectric element. The value of the dielectric constant was estimated to be about 4.4. For the patch element, copper adhesive tape would be attached to the G-10 board. Using the square model of the patch antenna, the optimized length of each side of the conductor was .47 wavelengths (figure 6).

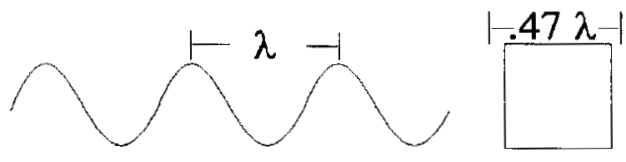

Figure 6: Relation Between Wavelength and Patch Size 
At this size, resonance would occur, and the efficiency of transmission would be improved. The wavelength of the signal was dependent on the constant of the dielectric insulator. In air, $\lambda=c / f$; $c=3 * 10^{\wedge} 8 \mathrm{~m} / \mathrm{s}$. In a solid object, however, $\lambda=U / f ; \quad U=c / \sqrt{ } \mu_{\mathrm{r}}$. Using these equations, the dimensions of an antenna were calculated for an optimized transmission at $2250.5 \mathrm{MHz}$ (figure 7). The feed point was connected in the center of the patch because it was not yet understood how to determine the impedance at a specified position on the patch. Next, the patch was built and tested with the results as found in table 2 .

\begin{tabular}{|c|c|}
\hline Length of Side & $33 \mathrm{~mm}$ \\
\hline $\begin{array}{c}\text { Dielectric Material } \\
\text { and Constant }\end{array}$ & $\begin{array}{c}\text { G-10 Epoxy } \\
\text { Board } \mu_{\mathrm{r}}=4.4\end{array}$ \\
\hline $\begin{array}{c}\text { Feed Line Distance } \\
\text { From Side }\end{array}$ & $16.5 \mathrm{~mm}$ \\
\hline $\begin{array}{c}\text { Standing Wave Ratio } \\
\text { (SWR) }\end{array}$ & 3.23 \\
\hline Return Loss & $-5.6 \mathrm{~dB}$ \\
\hline $\begin{array}{c}\text { Antenna Load } \\
\text { Impedance }\end{array}$ & $15.4+\mathrm{j} 10.2 \Omega$ \\
\hline $\begin{array}{c}\text { Transmission } \\
\text { Strength }\end{array}$ & $-36.9 \mathrm{~dB}$ \\
\hline
\end{tabular}

\section{Table 2: First Patch Antenna} Measurements@2250.5 MHz

This first patch antenna exhibited many improvements over the coaxial antenna link. Firstly, the SWR was reduced from 4.1 to 3.23 , a $21.2 \%$ reduction. As a result of a smaller SWR, less energy was lost from reflection resulting in a smaller return loss. These improvements resulted from load impedance being more closely matched to the optimal $50 \Omega$. Transmission loss was reduced significantly as a result of the changes; however, it was still far below satisfactory levels. To further improve the matching of antennas, impedance of the antenna load needed to be increased, this could be achieve by moving the feed line connection toward one side. In the first antenna, the connection was placed in the center of the patch; this was not

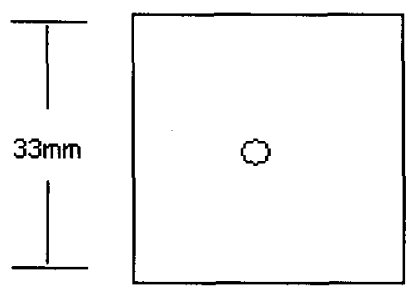

Figure 7: First Patch Design

the optimal position. The line needed adjustment to improve overall performance. In addition, the peak transmission rate was not at the desired $2250.5 \mathrm{MHz}$; rather, it was at a higher frequency. In order to adjust the patch so that the resonating frequency would be $2250.5 \mathrm{MHz}$, the patch size needed to be increased.

In the second test patch the length of each side was increased to $36 \mathrm{~mm}$ to decrease the resonating frequency. The feed point was moved closer to the side of the patch to an arbitrarily chosen $8 \mathrm{~mm}$ from the side (figure 8 ). The following measurements were recorded in table 3.

Once again, this new design was a vast improvement over the previous attempts. Better-matched impedance of the load resulted in a lower SWR of 1.85, a $41.6 \%$ increase over the previous attempt. Consequently, return loss was reduced to $-10.5 \mathrm{~dB}$. The transmission signal was much stronger than in previous attempts. Overcompensation was made in adjusting the antenna size 
to reduce the resonant frequency. The new resonating frequency was $2100 \mathrm{MHz}$, lower than the desired $2250.5 \mathrm{MHz}$.

\begin{tabular}{|c|c|}
\hline Length of Side & $36 \mathrm{~mm}$ \\
\hline $\begin{array}{c}\text { Feed Line Distance } \\
\text { From Side }\end{array}$ & $8 \mathrm{~mm}$ \\
\hline $\begin{array}{c}\text { Standing Wave Ratio } \\
\text { (SWR) }\end{array}$ & 1.9 \\
\hline Return Loss & $-10.5 \mathrm{~dB}$ \\
\hline $\begin{array}{c}\text { Antenna Load } \\
\text { Impedance }\end{array}$ & $28.7-\mathrm{j} 10.3 \Omega$ \\
\hline $\begin{array}{c}\text { Transmission } \\
\text { Strength }\end{array}$ & $-9.3 \mathrm{~dB}$ \\
\hline
\end{tabular}

Table 3: Second Patch Antenna Measurements@2250.5 MHz

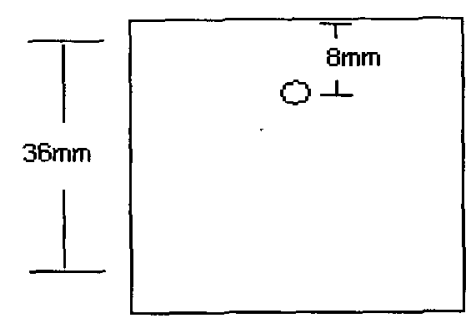

\section{Figure 8: Second Patch Attempt}

The improvements in the second antenna greatly reduced transmission loss. A slight reduction in the size of the patch would increase the resonating frequency to the desired transmission frequency. Furthermore, an adjustment in the feed point location would better match the load to the line, and reduce return loss.

In the third patch antenna design using the epoxy dielectric, the above stated modifications were made. Patch size was reduced, albeit slightly, to adjust the resonating frequency of the antenna. The side length was reduced to $35.5 \mathrm{~mm}$.
Also the feed point was moved closer the edge of the patch in an attempt to better match the load impedance to the transmission line.

The third attempt was an improvement over the previous patch designs. In this design, the size was very close to the desired resonating frequency improving the efficiency of transmission. The feed point of the antenna was also improved from that in the previous design. The SWR decreased from 1.85 to 1.57 , a $15 \%$ improvement, resulting in a smaller return loss. This decreased loss was a result of better matching between the antenna load and transmission line.

\begin{tabular}{|c|c|}
\hline Length of Side & $35.5 \mathrm{~mm}$ \\
\hline $\begin{array}{c}\text { Feed Line Distance } \\
\text { From Side }\end{array}$ & $8 \mathrm{~mm}$ \\
\hline $\begin{array}{c}\text { Standing Wave Ratio } \\
\text { (SWR) }\end{array}$ & 1.57 \\
\hline Return Loss & $-13.00 \mathrm{~dB}$ \\
\hline $\begin{array}{c}\text { Antenna Load } \\
\text { Impedance }\end{array}$ & $32.6-\mathrm{j} 7 \Omega$ \\
\hline $\begin{array}{c}\text { Transmission } \\
\text { Strength }\end{array}$ & $-4.61 \mathrm{~dB}$ \\
\hline Peak Frequency & $2200 \mathrm{MHz}$ \\
\hline
\end{tabular}

Table 4: Third Patch Antenna Measurements@2250.5 MHz

It was not known initially how the thickness of the dielectric would affect the efficiency of the antenna. It was necessary that thickness exceed $.01 \lambda$, but no other relationship was known concerning the patch efficiency and dielectric thickness. After the first few experiments, a spreadsheet calculator was made to improve the designing of the antenna (figure 9). Along with the formulas used previously, the file included a method for calculating the $50 \Omega$ impedance point. This would allow 
for better matching the load to the transmission line. The program placed the $50 \Omega$ spot at $.078 \lambda$ from the center. This analysis tool was used in the next design of the antenna.

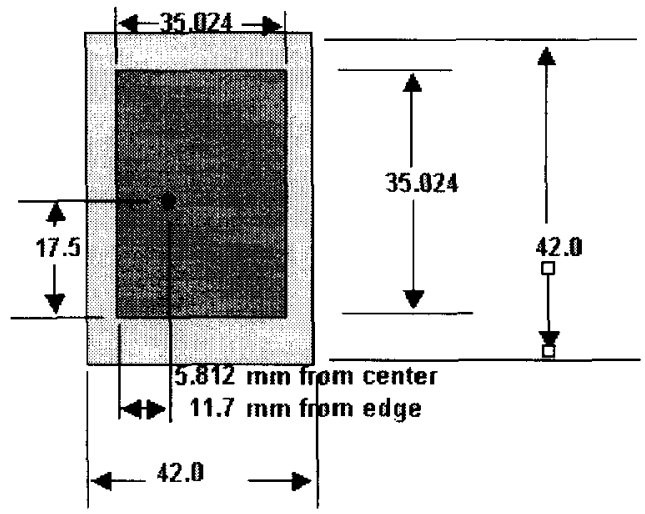

Figure 9: Output of the Spreadsheet Calculator

The subsequent antenna design using the spreadsheet calculator to do calculations did not yield expected improvements. Although the antenna load was matched very well against the transmission line, the efficiency of the antenna was reduced. It was observed, however, that the paper transmission barrier actually had an affect upon both the load resistance and optimal patch size. The paper adjusted the impedance of the load so that it was no longer matched to the transmission line.

When the paper was removed, the transmission was optimized at the specified frequency. Thus it was concluded that the equations used in the excel file assumed that the transmission medium was air. This faulty assumption neglected a variable from the calculations and resulted in an incorrect design. It was determined that the third

\begin{tabular}{|c|c|}
\hline Length of Side & $35 \mathrm{~mm}$ \\
\hline $\begin{array}{c}\text { Feed Line Distance } \\
\text { From Side }\end{array}$ & $11.7 \mathrm{~mm}$ \\
\hline $\begin{array}{c}\text { Standing Wave Ratio } \\
\text { (SWR) }\end{array}$ & 1.7 \\
\hline Return Loss & $-11.3 \mathrm{~dB}$ \\
\hline $\begin{array}{c}\text { Antenna Load } \\
\text { Impedance }\end{array}$ & $52-\mathrm{j} 28.4 \Omega$ \\
\hline $\begin{array}{c}\text { Transmission } \\
\text { Strength }\end{array}$ & $-7.2 \mathrm{~dB}$ \\
\hline
\end{tabular}

Table 5: Excel Patch Antenna Measurements $@ 2250.5 \mathrm{MHz}$

patch design was the best, and that the transmission strength was at an acceptable level. Unfortunately, it was learned at this time that the $4 \mathrm{~mm}$ thick epoxy board, which was used for the dielectric, was too thick and would not be acceptable. Use of this dielectric would demand that too much of the aluminum be milled out. Therefore, the antenna needed to be redesigned using a new dielectric.

This first stage of antenna design had many promising results. It revealed that the patch antenna was a viable method for accomplishing the specified tasks. The third antenna had been able to function at a satisfactory level of efficiency. The design had yielded a maximum strength of $-4.61 \mathrm{~dB}$, which maintained a sufficiently strong signal. Furthermore, the antenna in this stage demonstrated a bandwidth of about $150 \mathrm{MHz}$. Within the bandwidth, all values of return loss measured less than $-3 \mathrm{~dB}$ meaning that within these values, less than half of the signal power was lost from reflection (figure 10). Thus the width of the spike seen in the return loss measurement was relatively broad. This bandwidth allowed for a margin of difference between the transmitting and receiving antennas. Even if the resonant 
frequencies of the two antennas were not exact, the broad bandwidth increased the likelihood that the region of the spike would overlap for the two antennas. Where the two spike regions overlapped was the region where transmission was strongest. Thus, the broad bandwidth of this antenna was advantageous.

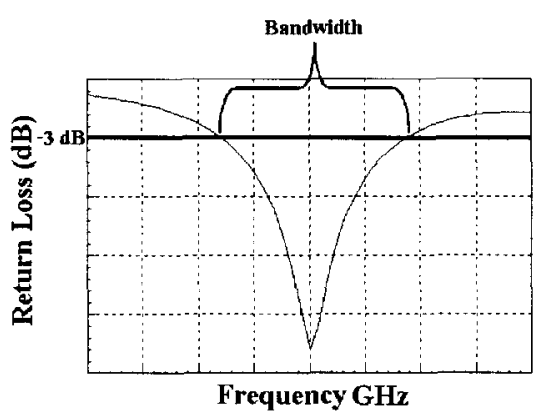

Figure 10: Bandwidth of the Antenna

These first experiments also revealed some problems with the G-10 antenna design as well as with the methods of measurement. The primary concern with this antenna was its size. Not only was the antenna too thick to be set within a milled out plate, but the thickness also produced an undesirable complex impedance. A thinner dielectric would resolve the size issue. The effects of the thinner dielectric upon the complex impedance were uncertain. Further experimentation would be more accurate at measuring the antenna performance. In these first experiments, the impedances of the antennas were measured before the plates were placed upon the stack of paper. It was not known initially that the paper would affect the impedance of the antenna. Therefore, the recorded impedances, SWR, and return loss were not the same values seen by the system when the transmission strength was being measured. This explained why the third patch had such good performance even though the line and load were not matched very well. When the antenna was placed above the paper, the impedance was adjusted and better matched to the transmission line. In the subsequent experiments, the impedance of the antenna was measured after the antenna was already in place with the paper.

Finally, some useful guidelines were discovered during the first experiments that would be helpful for further designs. Since the optimal length of the antenna side was dependent on the frequency, there was a correlation between a change in frequency and a change in optimal length. Higher frequencies have smaller wavelengths. As a result, higher frequencies have a smaller optimal side length. As side length increased, resonant frequency decreased. Also, as the feed line connection point moved away from the center of the patch, the impedance increased. These properties of patch antenna were useful to note and helpful for making adjustments in later designs.

\section{Thin Film Dielectric}

For the next set of experiments it was determined that a 1-mil thick kapton film dielectric (constant of 3.98) could be used. This thinner dielectric was hoped to minimize imaginary impedance of the antenna. Also the thinner antenna could easily be set within the milled out aluminum plate. One layer of the kapton was insufficient to satisfy the specification that the dielectric be at least $.01 \lambda$ in thickness. Therefore, several layers were used. 
Using the spreadsheet calculator to assist in computing a rough geometry for the antenna, it was determined how many layers would be needed. The dimensions given by the spreadsheet calculator were faulty because the program did not take into consideration the dielectric constant of the medium of transmission. The resonant frequency was not close to the desired 2205.5 MHz. Nevertheless, it was determined that a three-layer dielectric would be sufficiently thick. Using this thickness, the return loss was a minimized $-20 \mathrm{~dB}$ at a frequency of $3.63 \mathrm{GHz}$. With a dielectric as thick as 20 layers, the return loss was reduced to $-36 \mathrm{~dB}$ at $3.1 \mathrm{GHz}$. Although performance was better with more layers, both efficiency and size needed to be considered, and the return loss using three layers was acceptable. Unfortunately, using the three-layer antenna, with dimensions provided by the spreadsheet calculator, was not effective, and new dimensions for the patch element were needed.

\begin{tabular}{|c|c|}
\hline Length of Side & $32 \mathrm{~mm}$ \\
\hline $\begin{array}{l}\text { Dielectric Material } \\
\text { and Constant }\end{array}$ & $\begin{array}{c}\text { Kapton Sheet } \mu_{\mathrm{r}}= \\
3.98\end{array}$ \\
\hline $\begin{array}{l}\text { Feed Line Distance } \\
\text { From Side }\end{array}$ & $8 \mathrm{~mm}$ \\
\hline Resonant Frequency & $3.6 \mathrm{GHz}$ \\
\hline $\begin{array}{c}\text { Return Loss at } \\
\text { Resonant Frequency }\end{array}$ & $-20 \mathrm{~dB}$ \\
\hline
\end{tabular}

Table 6: Kapton Dielectric Antenna Measurements

To improve the design, the patch side length was increased so that it would have a lower resonant frequency. The side length was increased to $50 \mathrm{~mm}$. Also, to better match impedances the feed point was moved from $8 \mathrm{~mm}$ to $13 \mathrm{~mm}$ from the side. The design was tested with results found in table 7 . Maximum efficiency was at $2.3 \mathrm{GHz}$. At this level, transmission strength was $-15.0 \mathrm{~dB}$.

\begin{tabular}{|c|c|}
\hline Length of Side & $50 \mathrm{~mm}$ \\
\hline $\begin{array}{c}\text { Dielectric Material } \\
\text { and Constant }\end{array}$ & $\begin{array}{c}\text { Kapton Sheet } \mu_{\mathrm{r}} \\
=3.98\end{array}$ \\
\hline $\begin{array}{c}\text { Feed Line Distance } \\
\text { From Side }\end{array}$ & $13 \mathrm{~mm}$ \\
\hline $\begin{array}{c}\text { Standing Wave Ratio } \\
\text { (SWR) }\end{array}$ & 3.2 \\
\hline Return Loss & $-8.1 \mathrm{~dB}$ \\
\hline $\begin{array}{c}\text { Antenna Load } \\
\text { Impedance }\end{array}$ & $31+\mathrm{j} 4 \Omega$ \\
\hline $\begin{array}{c}\text { Transmission } \\
\text { Strength }\end{array}$ & $-15.0 \mathrm{~dB}$ \\
\hline Peak Frequency & $2.3 \mathrm{GHz}$ \\
\hline
\end{tabular}

Table 7: Kapton Dielectric Antenna Measurements@2205.5 MHz

After this test, the kapton was no longer used. The kapton was a flimsy material, and using several layers it was difficult to make flat, sturdy patches. When soldering the feed line to the copper patch, the kapton inevitably melted and disfigured from the heat. Consequently, results from the patches were inconsistent. If the patch was agitated between measurements, different results were measured making precision difficult. Also, the bandwidth of the resonant frequency was limited to about $60 \mathrm{MHz}$, making consistency and precision necessary considerations. In addition, imaginary impedance had not been satisfactorily reduced. A net capacitive effect had been replaced with a smaller net inductive effect. This set of experiments revealed a connection between dielectric width and bandwidth. The thinner dielectric resulted in a narrower bandwidth requiring too much precision. 


\section{New Design Strategy}

For the next set of experiments, a slightly thicker dielectric was desired. A dielectric thinner than the first G-10 board and thicker than the kapton film would still meet size constraints, and was hoped to further reduce complex effects upon antenna impedance by better balancing inductive and capacitive effects within the antenna. The thicker dielectric would be less flimsy and would also have a moderate bandwidth. In addition, a new approach to the problem was used. In the following experiments, the connector pin was soldered in the center edge of the width side (figure 11). Placing the pin in this position would allow for slight adjustments in the antenna design to improve performance. The patch would no longer be square. With the connector to one side, adjusting patch width was used to vary impedance. It was found that decreasing the length would increase the resonant frequency and decreasing the width would increase the input impedance. This statement is just a general rule, however, as both width and length had effects on both resonant frequency and input impedance. These rules were used in an attempt to finetune the antenna.

Another change in this stage of the experiment was the use of a new CAD tool called MPAC 0.2 .2 (figure 12). This program used more precise equations than the spreadsheet calculator did to compute an appropriate geometry. Unfortunately, this program, like the spreadsheet calculator did not take the paper dielectric into account in designing the antenna. Despite this shortcoming, the program was helpful in getting a starting point from which the antenna could be trimmed and tuned. It was also useful in analyzing how one variable of input would affect the outputs. Finally, the program code could be modified to include addition variables once the affects of these variables were understood.

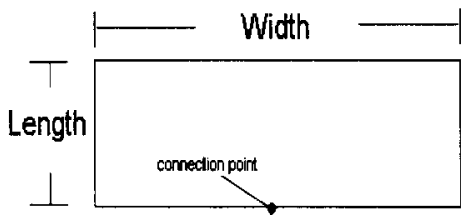

\section{Figure 11: New Design Strategy}

At this time, more attention was placed on optimizing a single antenna before measuring the performance of a link. Previous experiments had yielded a satisfactory links using antennas with small return loss. It was believed that if an efficient antenna were designed using these materials, a satisfactory link would again be achieved. At this stage of the project, the goal was to design an antenna with the spike occurring at the specified $2250.5 \mathrm{MHz}$. It was believed also, that if the antenna were well matched to approximately $50 \Omega$, the resulting return loss would be between -20 and $-30 \mathrm{~dB}$. Additional variables were also considered at this time to reduce the guessing involved in choosing dimensions for the antenna. Previously, it was discovered that the paper dielectric between the plates had the effect of altering the resonant frequency of the antenna. It was now revealed that increased force on the back of the aluminum plate compressed the paper and resulted in even further variation of the resonant frequency. The antenna to be designed was to operate 
within specifications with the paper in place and compressed to best represent specified conditions. Understanding the effects of the paper dielectric, a new design was attempted. The initial size of this antenna was $100 \mathrm{~mm} \mathrm{X} 50 \mathrm{~mm}$ and the connector was placed along the long edge, $50 \mathrm{~mm}$ from each side. This antenna was not efficient. The resonant frequency was too low. To compensate, the length was trimmed slightly, increasing the resonant frequency. When the resonant frequency was close to the desired value, the width was reduced to increase impedance of the antenna to $50 \Omega$. Finally, a tall spike centered at $2.2505 \mathrm{GHz}$ was achieved. The dimensions of the antenna were $84.5 \mathrm{~mm}$ X $34 \mathrm{~mm}$ with the connection in the center of the long side. Measurements with this geometry were as follows recorded in table 8 .

\begin{tabular}{|c|c|}
\hline Dimensions & $84.5 \mathrm{~mm}$ X 34 mm \\
\hline $\begin{array}{c}\text { Standing Wave Ratio } \\
\text { (SWR) }\end{array}$ & 1.03 \\
\hline Return Loss & $-22.4 \mathrm{~dB}$ \\
\hline $\begin{array}{c}\text { Antenna Load } \\
\text { Impedance }\end{array}$ & $50.7-\mathrm{j} 1.7 \Omega$ \\
\hline Peak Frequency & $2.2505 \mathrm{GHz}$ \\
\hline Bandwidth & $92 \mathrm{MHz}$ \\
\hline $\begin{array}{c}\text { Transmission } \\
\text { Strength }\end{array}$ & $-7.7 \mathrm{~dB}$ \\
\hline
\end{tabular}

\section{Table 8: Thin G-10 Antenna Measurements}

This final test antenna performed as anticipated. Tuning the antenna resulted in a closely matched antenna load. Complex effects were reduced significantly from those in the original G-10 antenna. Minimal capacitance and tunable impedance resulted in a low SWR and return loss within predicted

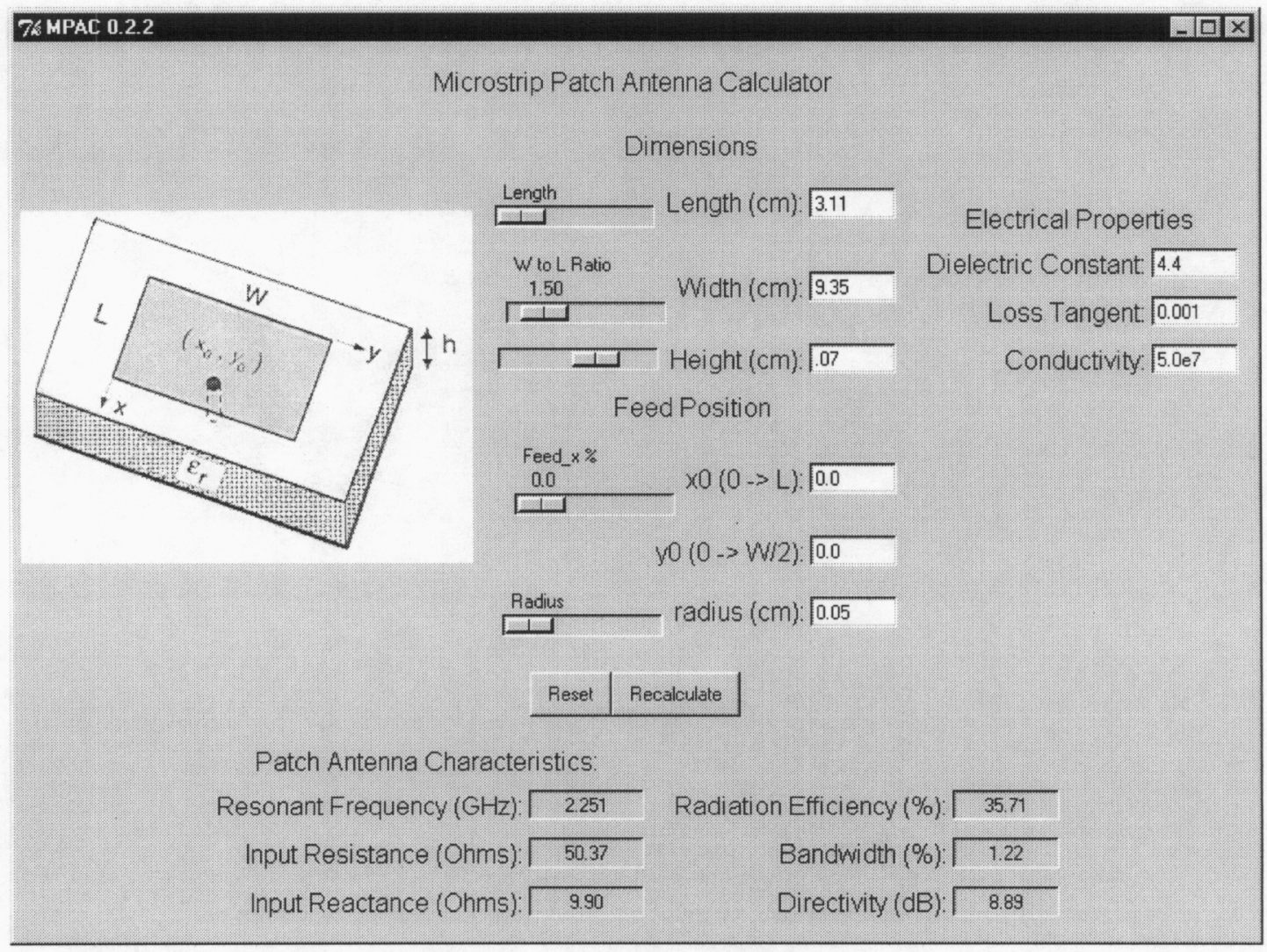

Figure 12: MPAC Antenna Design Program 
levels. This model also met all of the size constraints. The bandwidth was within predicted moderate, narrower than that of the original G-10 antenna, yet broader than that in the kapton film models. This bandwidth still required some precision in fabrication. This third set of experiments did not yield a transmission performance as good as the first set of experiments had. This was most likely due to the narrower bandwidth. This narrower bandwidth caused that the region of overlap between the resonant area of the transmitting and receiving antennas was less tolerant of variance between the two antennas. The other main issue of concern was the inconsistency of the medium of transmission.

\section{Future Recommendations}

For future experiments, the pressure placed on the plates needs to be standardized as variances in pressure distort results. Even better would be to replace the paper in the testing apparatus with a consistently shaped sheet of plastic. Also, using metal plates with milled out slots for the patch antenna would allow consideration of any effects resulting from the antenna being set within the large reflector plate. Finally, in future experiments, MPAC should be adjusted to take into account the dielectric constant of the medium of transmission in calculating the antenna characteristics. These adjustments would reduce the amount of tuning made to match the antenna impedance to the line. Also, these improvements would allow for quick calculations of antenna dimensions using different mediums of transmission.

\section{Conclusions}

These experiments were very useful in the design of the wireless link. Not only did they demonstrate that patch antennas were a good approach to the specified application, but they established a foundation of understanding for future patch antenna design. 